Behavioural Sciences | Joanna Tully \& Janine Rowse

\section{Technology-facilitated sexual assault of children and adolescents}

A retrospective audit

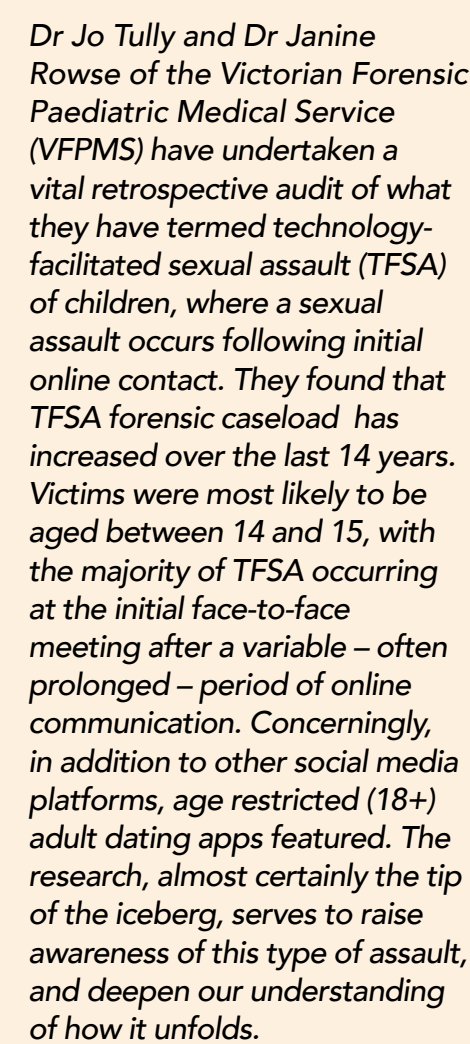

the Victorian Forensic Paediatric the Victorian Forensic Paediat
Medical Service (VFPMS) were concerned about the increasing number of children presenting for a that their perpetrators had contacted them online. There was no existing data available to them describing the prevalence or characteristics of what the researchers termed technologyfacilitated sexual assault (TFSA) of children. This absence of data, coupled with the concerning frequency of these cases, led the researchers to undertake a retrospective audit of the previous 14 years of case notes from their service. Their study has yielded some highly concerning results, and has highlighted the need to finesse and deepen our understanding of the relationship between online technologies and sex assault. The researchers highlight, start, and there remains a huge amount of sensitive work to be done in this area. of how it unfolds. The VFPN, which is based in a which involves addressing any medical

Dating-app-facilitated sexual assault
THE BROADER CONTEXT provides advice aran setting, under 18 years of agr for children allegation of sexual assault. The service undertakes a thorough examination needs, examining the body for any injuries, and collecting forensic evidence. Anecdotal evidence strongly suggested that there has been an increase in allegations where contact with the perpetrator was initiated through online social media. of adults has recently been receiving considerable media attention in Australia and in numerous other areas around the world, where the is extensive use of online social concern thertic the high level of information. There has been even less research undertaken related to TFSA in children who are more vulnerable to this type of offence due in part to their limited ability to risk assess. Parents are fearful for their children, particularly teenagers, for whom online communications have become one of the main ways in which they socialise.

Connecting via social networking platforms has become mainstream. Access to a smart device is almost ubiquitous among young people. The popularity of plattorms used has evolved. Previously, Facebook more recently, more contemporary platforms such as YouTube, Snapchat Chd Instagram have become popula Children are also using dating apps, communicate and connect.

Given this background and the anecdotal evidence from the VFPMS, Tully and Rowse undertook a retrospective review of the records of children presenting for forensic examinations, to further understand this phenomenon.

\section{THE METHOD}

Tully and Rowse conducted a 14-year retrospective audit of their case notes. They reviewed all the medical notes of the children, aged 12 to 17 years, who accessed the VTMS for a forensic medical examination after an alleged of its kind all cases wist spectic study which technology was used as the first method of contact between victim and offender (technology facilitated sexual assault - TFSA).

The audit had two aims: to facilitate understanding of the characteristics of TFSA, and

to increase the

understanding

of prevalence.

The data set was

organised into two

groups; one 2007

to 2013 and the other 2014 to 2020 , so chat chang win these time periods could be ascertained.

\section{THE FINDINGS}

\section{Prevalence of TFSA}

the first half of the study the 2007-2013 group, it was found that $4 \%$ of alleged sexual assaults of children seen by the service were technology-facilitated. In the second half of the study, the 2014-2020 group, $14 \%$ of sexual assaults were technology-facilitated. In the last year of the study, 2019-2020, this had risen to $19 \%$. These findings vindicated the researchers' initial fear that these types of sexual assault were - Chaning more common.

- Characteristics of TFSA

The researchers found that $89 \%$ of victims .

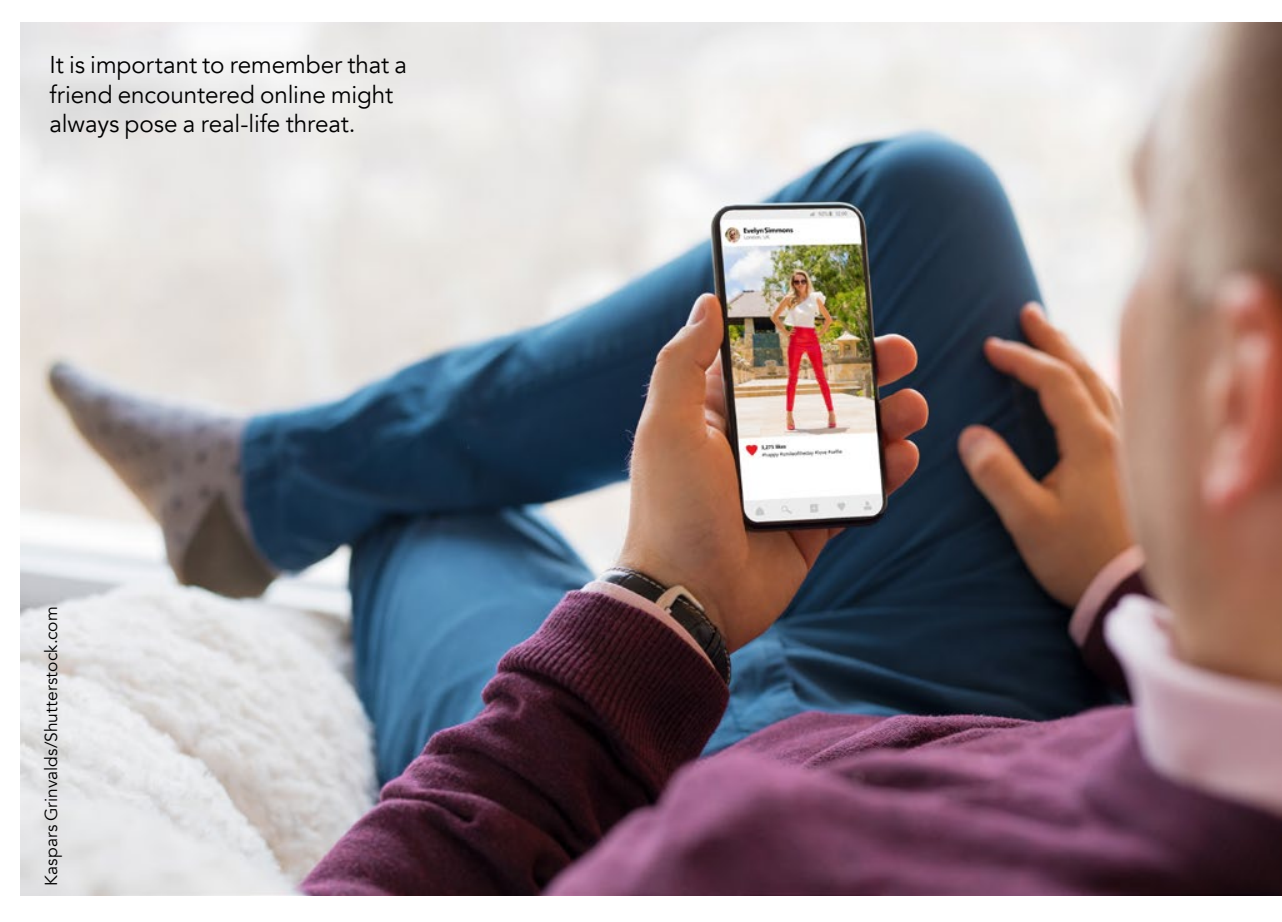

victims of TFSA was 15, with the majority face-to-face. More research is planned of victims aged 15 or under. to discern the particular conversationa

The majority of TFSA occurred at the first face-to-face encounter, but the length of online communication preceding the assault varied. Some between first contact and initial meeting.

In approximately one third of TFSA cases, the assault occurred at the alleged gardless of the duration or nature $\quad \begin{aligned} & \text { and in another third, } \\ & \text { the TFSA occurred in a }\end{aligned}$ f online contact, an online friend public space (for example is a real-life stranger. interactions were less than 24 hours between online contact to face-toface meeting, whereas others had weeks and up to a year before meeting More children are using dating apps.
at younger ages.

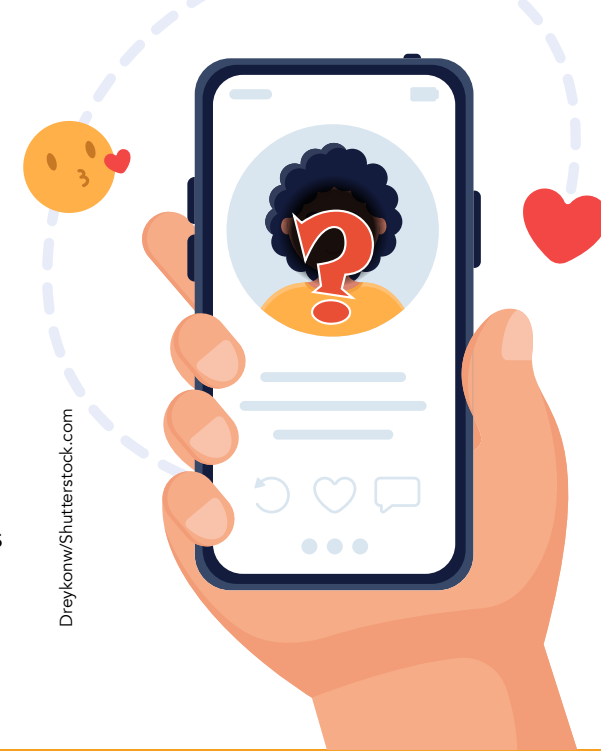
parks or public toilets). The overwhelming majority of sexual assault allegations involved penetration of the vagina, mouth and/or anus. Three undertaken without the use of a condon, putting the child at even further risk f harm through sexully transmitted diseases and pregnancy.

Whilst all the alleged offenders were male, their ages varied; most were more than two years older (up to 26 years older) but many were of the same peer group as the victim. In soffenders had deliberately misled the victim about their age.

- Platforms for TFS The social media plattorm used to enac TFSA changed over the years of the platforms Between 2007 and 2013 Facebook was the 20 oni 2013 , associated with TFSA, but between 2014 
and 2016, dating apps (designed for adult use) began to be identified in the audi. This chich pook a dramatic turn in 2017 TFSA cases (almost hast was associated with the app Snapchat.

Snapchat is a popular platform used by children 13 and over, facilitating time-limited image sharing, which is generally viewed by parents or carers as a risky app (the impermanence of the images, perhaps, contributing to the idea that the app is generally harmless. 2017 to 2020 saw a decrease in the proportion of Facebook TFSA, and an increase in the proportion of TFS identified as being facilitated throug dating apps (almost a third.

\section{THE KEY MESSAGES}

\section{AND CONCLUSIONS}

Harm to children's physical and menta

health results from both online and

'offline' sexual abuse, but this import

research highlights how the one

can easily and increasingly facilitate

the other. Greater public awareness

is needed to reduce the stigma of

online contact, as our understanding of the social politics of online discourse particularly pertaining to dating apps is still in its infancy.

Ultimately, TFSA is increasing. Given the globally unprecedented accessibility of the online space to children, there is a greater need than ever before to be
aware that this can happen to anyone's

child. Where previously parents could exert a certain level of control over who too easy for childen to through social media platforms and adu dating apps. Although online grooming of children has been comprehensively studied, Tully and Rowse's research provides further insight specifically into the characteristics of offline contact sexual offences following meeting online. This research should pave the way to a deeper and more nuanced understandr of a pernicious consequence of children's online discourse.

Social media companies have responsibility to ensure the safety of child users.

Tully and Rowse call for greater and their senior executives in protecting particularly in comparison to nonchildren in the online space. Urgent that sexual harm online is occurring education of children in reframing of at increasingly younger ages, future online stranger danger is needed: analysis will be extended to include regardless of the duration or nature of online contact, an online friend is a real-life stranger. Public health promotion messages - such as 'it's OK to say no' to image sharing, or 'no bedrooms or bathrooms' for digital technology use - should be widely promoted. Health professionals should also be encouraged tonormalise a broader conversation about children's online activity.

There is known to be a significant underreporting of child sexual assault, and there are possibly additional it is alnost certing TFSA. Worryingly, greater public awareness of stigma, normalise asking about online activities, promote public health messages related to online stranger platform providers.

Further analysis and ongoing data collection is underway, to focus on developing a deeper understanding younger age groups.

A collaboration has been developed with the Australian Childhood Foundation, to work towards . resources, aimed at both children and parents, related to online safety. The more this issue enters the public conversation, the less obscure these risks will become, and the greater chance we will have
safeguarding children online.

\section{Behind the Research 是 Dr Joanna Tully \\ Dr Janine Rowse}

Research Objectives

Dr Jo Tully and Dr Janine Rowse have undertaken a retrospective audit of technology-facilitated sexual assaut (TFSA) of children.

\section{Detail}

Address

Victorian Forensic Paediatric Medical Service Monash Children's Hospital

246 Clayton Road

Australia

Bio

Dr Jo Tully is a forensic paediatrician and Deputy Director of the Victorian Forensic Paediatric Medical Service (VFPMS), Australia. Dr Janine Rowse is a clinical forensic medicine practioner at the VRMS and the Victorian Institute of Forensic Medicine (VIFM)

Collaborators

Department of Forensic Medicine, Monash University
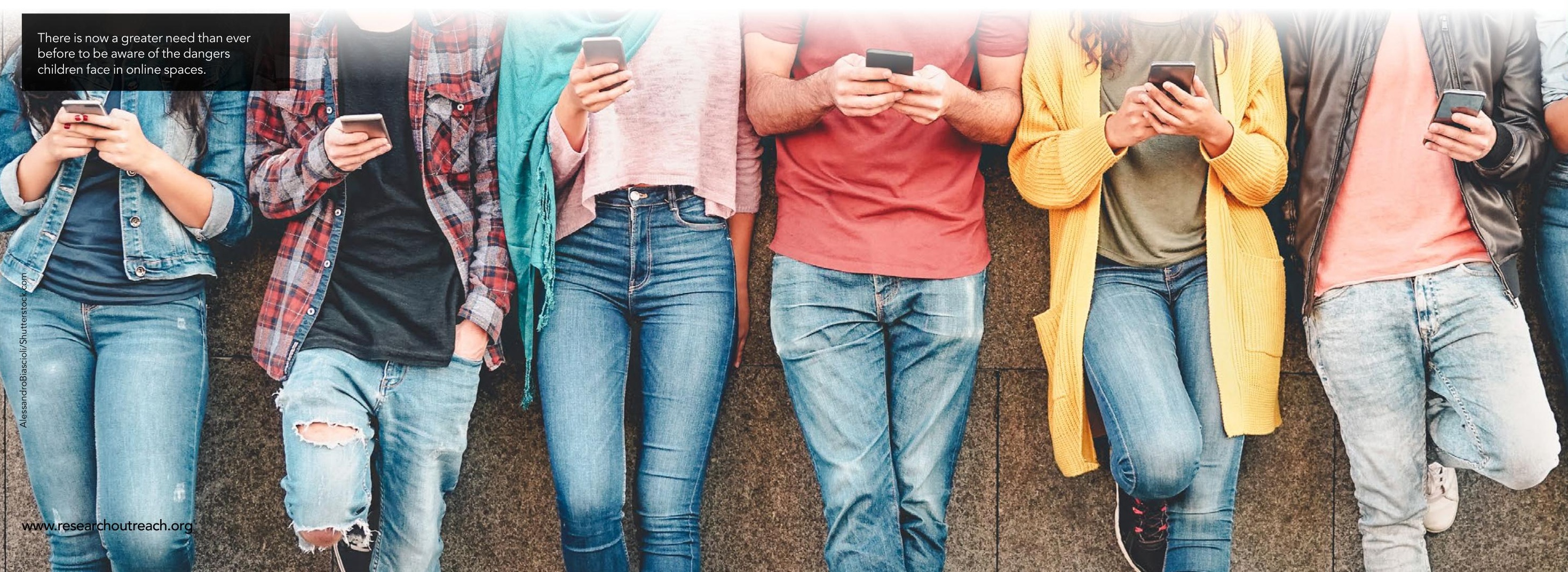

References

Rowse, J, Mullane, S, Bassed, R, Tully, J, (2021) Technologyfacilitated sexual assault in children and adolescents; is there a cause for concen? Founteen years of experience at a metropolitan forensic paediatric medical service, $J$
Paediatr Child Health. doi.org/10.1111/jpc.15724

\section{Personal Response}

To what extent do you now feel the onus is on social media companies to develop their own processes to prevent or to monitor this concerning emerging phenomena?

II Technology-facilitated sexual assault of children is occurring, and likely increasing. Social media companies have a high level of responsibility to ensure respond so emerging trends and acknowlenge the possibility of online and offline sexual harms.

Social media platforms, including apps intended for adult use, must increase their accountability and systems approaches to prevent children from analogous to controls over the sale of alcohol to min in many jurisdictions.

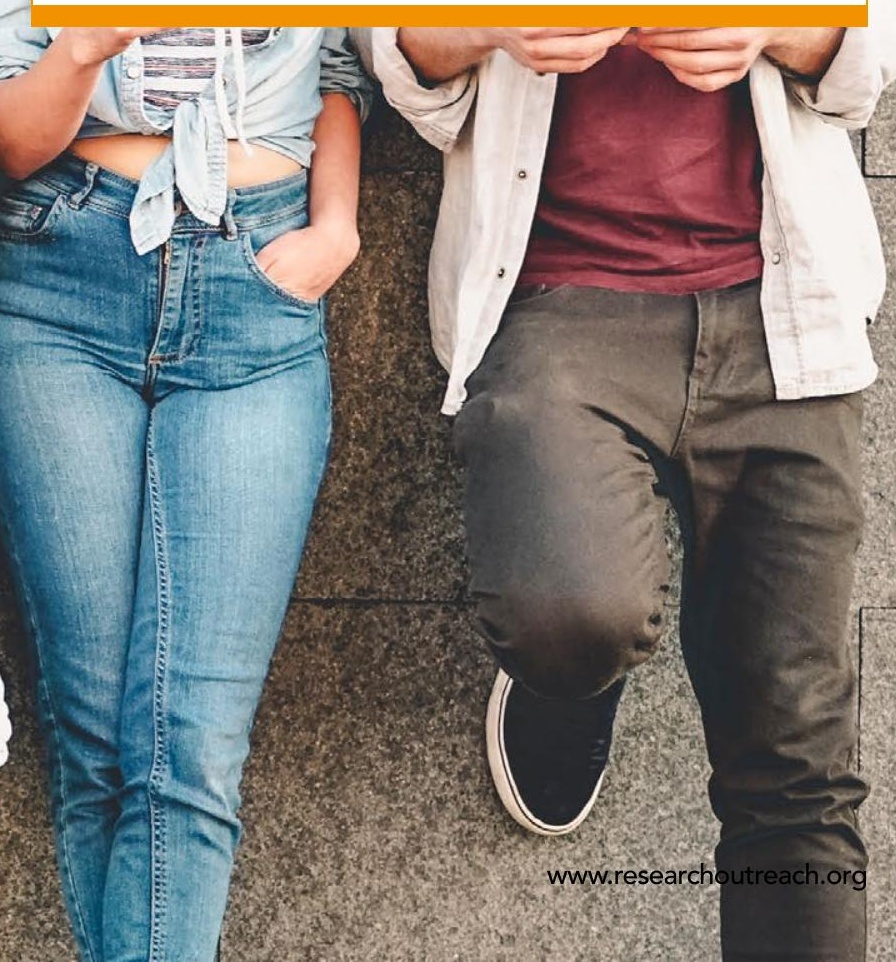

